\title{
Ferroelectric Polarization Induces Electric Double Layer Bistability in Electrolyte-Gated Field-Effect Transistors
}

Simone Fabiano, Xavier Crispin and Magnus Berggren

\section{Linköping University Post Print}

\section{Tweet}

N.B.: When citing this work, cite the original article.

Original Publication:

Simone Fabiano, Xavier Crispin and Magnus Berggren, Ferroelectric Polarization Induces Electric Double Layer Bistability in Electrolyte-Gated Field-Effect Transistors, 2014, ACS Applied Materials and Interfaces, (6), 1, 438-442.

http://dx.doi.org/10.1021/am404494h

Copyright: American Chemical Society http://pubs.acs.org/

Postprint available at: Linköping University Electronic Press http://urn.kb.se/resolve?urn=urn:nbn:se:liu:diva-104116 


\title{
Ferroelectric polarization induces electric double
}

\section{layer bistability in electrolyte-gated field-effect transistors}

\author{
Simone Fabiano, * Xavier Crispin, and Magnus Berggren
}

Organic Electronics, Department of Science and Technology, Linköping University, SE-601 74, Norrköping (Sweden) 
ABSTRACT: The dense surface charges expressed by a ferroelectric polymeric thin film induce ion displacement within a polyelectrolyte layer, and vice versa. This because the density of dipoles along the surface of the ferroelectric thin film and its polarization switching time matches that of the (Helmholtz) electric double layers formed at the ferroelectric/polyelectrolyte and polyelectrolyte/semiconductor interfaces. This combination of materials allows for introducing hysteresis effects in the capacitance of an electric double layer capacitor. The latter is advantageously used to control the charge accumulation in the semiconductor channel of an organic field-effect transistor. The resulting memory transistors can be written at a gate voltage of around $7 \mathrm{~V}$ and read out at a drain voltage as low as $50 \mathrm{mV}$. The technological implication of this large difference between write and read-out voltages lies in the non-destructive reading of this ferroelectric memory.

KEYWORDS: electrolytes, ferroelectric, $\mathrm{P}(\mathrm{VDF}-\mathrm{TrFE})$, electric double layer, memory, fieldeffect transistors

\section{Introduction}

Memory functionality is a prerequisite for many electronic applications in modern society. In fact, most of the envisaged electronic applications require non-volatile memory systems that can be programmed, erased and read-out electrically. ${ }^{1,2}$ Organic non-volatile memory devices based on ferroelectric polymers represent a promising approach towards the development of a low-cost technology that is possible to manufacture using common printing techniques. ${ }^{3}$ Ferroelectric polymers are especially attractive as the active medium for data storage, owing to their high flexibility, low cost and simple production protocol. ${ }^{4-6}$ Ferroelectric memories based on the 
copolymer poly(vinylidenefluoride-co-trifluoroethylene) (P(VDF-TrFE)) are of particular interest to the organic electronics community due to the excellent processability (i.e. low temperature and from solution). The ferroelectricity of this material stems from the molecular dipole moments, residing perpendicular to the $\mathrm{P}(\mathrm{VDF}-\mathrm{TrFE})$ backbone defined by the electronegativity difference between hydrogen-disubstituted carbon and fluorine-disubstituted carbons. The dipoles can be aligned collectively with the applied field by a rotation of the monomer groups in crystalline domains. The binary states of the memory device reflect two stable polarization states of the ferroelectric material that can be maintained even in the absence of an externally applied electric field. Switching between the two polarization directions occurs only above a certain threshold of the external electric field, which provides the non-volatile character. Organic ferroelectric field-effect transistors are technologically relevant because they can be addressed easily in an array of memory elements.

In the last years, several groups have demonstrated that solution processable solid polymer electrolytes can be used as gate insulator materials in transistors. ${ }^{7-9}$ In electrolyte-gated fieldeffect transistors (FETs) indeed, the polarization mechanism of such dielectrics involves the reorganization of the included ions forming two (Helmholtz) electric double layers, EDLs, on the microsecond time scale at the gate/electrolyte and electrolyte/semiconductor interfaces. Most of the applied gate-source bias drops across these EDLs. The charged sheets of the EDLs are separated by only a few Angstroms within the Helmholtz double layer, leading to extraordinary high transversal electric fields $\left(10^{9} \mathrm{~V} \mathrm{~m}^{-1}\right)$, established already at very low gate-source voltages. Thus, the high capacitance of the EDL capacitor enables low operating voltages, which are typically less than $1 \mathrm{~V}$. However, none has yet introduced a memory function in these lowvoltage FETs. 
Here, we demonstrate that a bi-layer comprising an electrolyte and a ferroelectric fluoropolymer can promote memory functionality when used as the gate dielectric in a polymer transistor. The electrolyte layer transfers efficiently the polarization state of the ferroelectric layer to the semiconductor channel via ion displacement and through the formation of an EDL at the electrolyte/semiconductor interface. We then combine the bistable polarization of the ferroelectric with the high capacitance of the EDLs to create low-readout voltage electrolytegated transistors, ideal for printed non-destructive memory elements.

\section{Results and discussion}

A polyelectrolyte comprising fixed anions and mobile cations is favorably utilized to separate the ferroelectric film from the semiconductor. The use of a polyelectrolyte rather than an electrolyte where both anions and cations are mobile, suppresses any undesired penetration of ions in the organic semiconductor (i.e. electrochemical doping). Hence, poly(vinylphosphonic acid-co-acrylic acid) (P(VPA-AA)) is chosen as the polyelectrolyte thin film whereas P(VDFTrFE) is used as a ferroelectric layer (Figure 1a). It is important to note that the polarization induced in the ferroelectric/polyelectrolyte bi-layer is not limited (in time and amplitude) by any of the materials since both ferroelectric and polyelectrolyte display similar surface charge density and time scale. $\mathrm{P}(\mathrm{VDF}-\mathrm{TrFE})$ shows indeed a remanent polarization as high as $10 \mu \mathrm{C} \mathrm{cm}^{-2}$, a value which is of the same order of magnitude as the EDL surface charge density in P(VPAAA). ${ }^{9}$ Moreover, the switching time for a $\mathrm{P}(\mathrm{VDF}-\mathrm{TrFE})$ layer is about $0.1 \mathrm{~ms}^{10}$ also of a similar order of magnitude as the EDL formation in P(VPA-AA). ${ }^{9}$ Regio-regular poly(3-hexylthiophene) (P3HT) is used as the semiconductor material because of its high field-effect mobility and low contact resistance when gold is used as source/drain electrodes. 


\section{a}

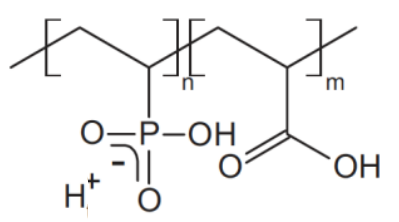

P(VPA-AA)

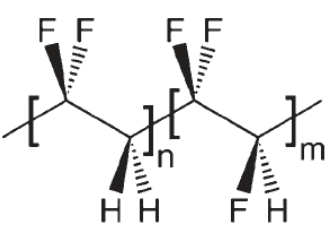

$P($ VDF-TrTE)

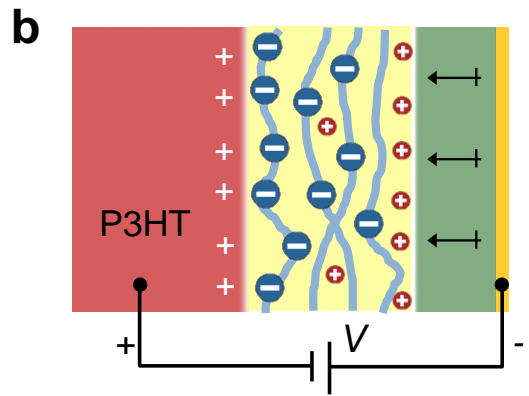

Figure 1. (a) Structure of the polyelectrolyte and ferroelectric copolymer. (b) Schematic illustration of the charge and ion distribution within the polyelectrolyte layer for a metainsulator-semiconductor structure.

The net surface charge density of the ferroelectric thin film can be induced to be either positive or negative depending on the direction of the applied polarizing electric field. It has been proposed that an EDL can be formed at the surface of an inorganic ferroelectric layer in contact with an electrolyte, leading to the electrostatic adhesion of charged particles. ${ }^{11,12}$ Recent studies have also shown that dipolar interactions identified as Coulomb attractions between hydrogen atoms in the PVDF chains and anions promote ferroelectric polarization in a PVDF-ionic liquid blend. ${ }^{13}$ Based on these concepts, we propose a mechanism of polarization across a ferroelectric/polyelectrolyte/organic semiconductor stack, which appears as a cross section of the ferroelectric electrolyte-gated FET presented below. The electrolyte is a polarizable medium that transfers the surface charge of the ferroelectric to the semiconductor surface, with only a minor potential drop across the bulk of the polyelectrolyte film. So, when a negative (positive) voltage is applied to the ferroelectric high enough to overcome the coercive voltage, that is, the minimum bias required to switch the full remanent polarization, two EDLs are established at the ferroelectric/polyelectrolyte and polyelectrolyte/semiconductor interfaces, respectively (Figure 1b). The first EDL is defined by the surface charges from the dipoles of the switched ferroelectric film balanced by the mobile counter ions of the electrolyte. The latter EDL is 
represented by the accumulated holes (electrons) residing along the outermost sheet along the organic semiconductor film balanced by charged polyelectrolyte chains (depleted of counter ions). The concept of EDLs induced by switching of the ferroelectric polarization is general and can be applied to $p$-type as well as $n$-type semiconducting materials.
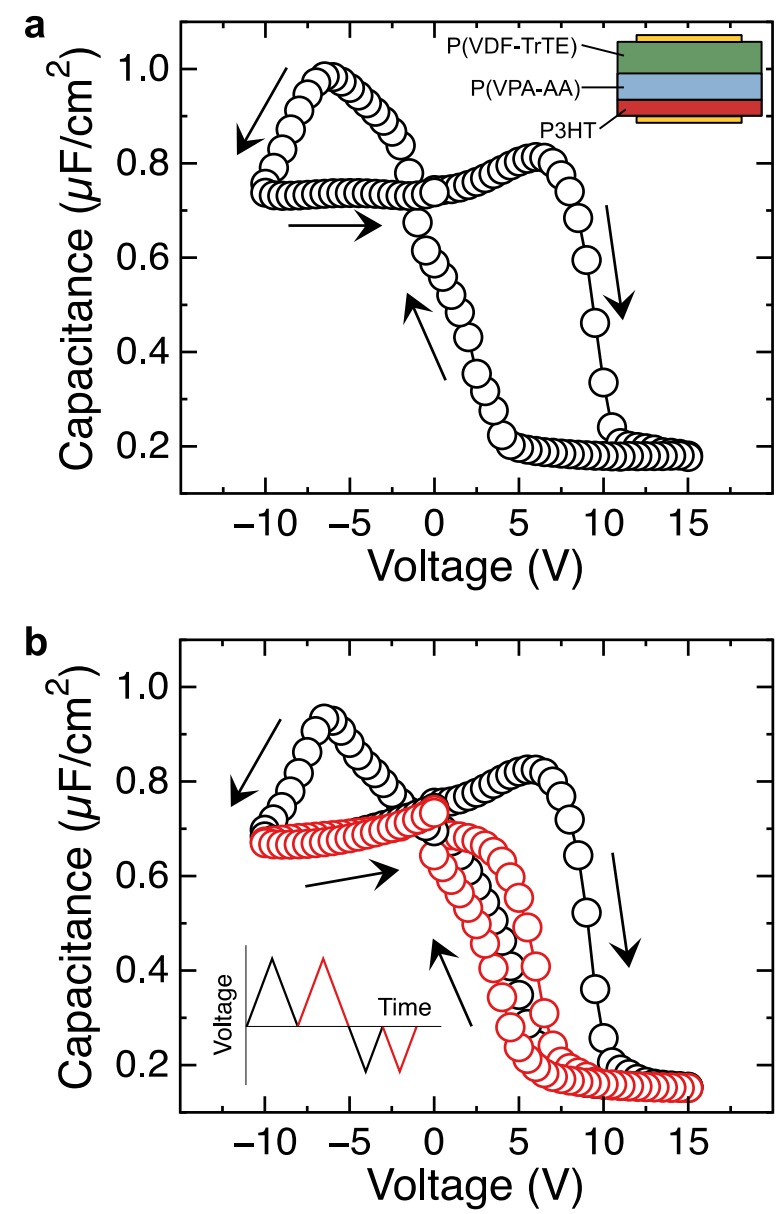

Figure 2. (a) Representative capacitance-voltage characteristic of a Au/P3HT/P(VPAAA)/P(VDF-TrFE)/Au-based MIS structure (see inset). (b) Programming the ferroelectric layer with a voltage sequence of the same polarity results in a loss of hysteresis corroborating the fact that ion migration is not the origin of the capacitance bistability. 
The capacitance-voltage $(C-V)$ characteristics of the ferroelectric/polyelectrolyte bi-layer were measured using a metal-insulator-semiconductor (MIS) structure consisting of a 140-nm-thick $\mathrm{P}(\mathrm{VDF}-\mathrm{TrFE})$ layer and a 120-nm-thick P(VPA-AA) layer sandwiched between a P3HT-coated $\mathrm{Au}$ bottom electrode and an evaporated $\mathrm{Au}$ top electrode (Figure 2a). At negative bias, i.e. negative voltage applied to the Au top electrode, holes are injected in P3HT (Ohmic contact ${ }^{14}$ ) and the ferroelectric layer is poled with the negative dipoles (F-terminated carbons) pointing towards the polyelectrolyte. The capacitance reaches up to $\sim 1 \mu \mathrm{F} \mathrm{cm}{ }^{-2}$, a value which compares with that of typical polyelectrolyte dielectrics. ${ }^{9}$ The resulting $C-V$ curve shows a marked nonlinearity and hysteresis and this behavior is also observed in P(VDF-TrFE)-only capacitors and stems from the bias-field-dependent permittivity of $\mathrm{P}(\mathrm{VDF}-\mathrm{TrFE}) .{ }^{15} \mathrm{~A}$ transition towards depletion occurs at a bias voltage of around $+7 \mathrm{~V}$, which is the expected coercive voltage for a 140-nm-thick $\mathrm{P}(\mathrm{VDF}-\mathrm{TrFE})$ layer $\left(E_{\mathrm{c}}=53 \mathrm{MV} \mathrm{m}^{-1}\right)$. The depletion capacitance at positive bias $(V>+10 \mathrm{~V})$ equals the estimated value derived from the reciprocal sum of the semiconductor $C_{\mathrm{s}}$ and ferroelectric/polyelectrolyte $C_{\mathrm{i}}$ layer capacitance and is here dominated by $C_{\mathrm{s}}$ (that is, $\sim 0.1$ $\mu \mathrm{F} \mathrm{cm}^{-2}$ for a 30-nm-thick P3HT layer assuming a dielectric constant of 3). The scan from $+15 \mathrm{~V}$ to $0 \mathrm{~V}$ has a transition point at around $+4 \mathrm{~V}$, which corresponds to the flat-band potential of the organic semiconductor. To stabilize the polarization at positive gate bias, the polyelectrolyte chains at the interface with $\mathrm{P}(\mathrm{VDF}-\mathrm{TrFE})$ should be negatively charged while electrons should accumulate in the P3HT layer. However, the electron current is negligible due to the large injection barrier at the Au source electrode-P3HT interface. Hence, the ferroelectric polarization cannot be compensated by an accumulation of electrons along the semiconductor interface. The ferroelectric film depolarizes to the pristine state, which leads to lack of remanent depletion. ${ }^{15}$ If two unipolar voltage loops are applied to the ferroelectric, only the first sweep will contribute to 
the switching of the ferroelectric dipoles. ${ }^{16}$ Programming of the ferroelectric layer with a voltage bias sequence of the same polarity results in a loss of hysteresis (Figure 2b). This suggests that ion migration is not the origin of the capacitance bistability switching in these devices.

The combination of ferroelectric bistability with semiconductivity allows for non-volatile memories with non-destructive read-out where the resistance and the polarization can be tuned independently. ${ }^{17}$ In this respect, ferroelectric field-effect transistors (FeFETs) are ideally suited for the achievement of memory technology, as they allow for a complete decoupling of the write and read-out functionalities. ${ }^{18,19}$ Top-gated polymer FETs were fabricated using the ferroelectric/polyelectrolyte bi-layer as the insulator and P3HT as the semiconductor channel (see Experimental methods for further details). Figure 3a-c shows the current-voltage characteristics of a representative device. The transistor operates in the accumulation mode where holes are the majority charge carriers. A typical transfer characteristic ( $I_{\mathrm{ds}}$ vs. $\left.V_{\mathrm{g}}\right)$, recorded in the linear regime at low source-drain bias $\left(V_{\mathrm{ds}}=-0.2 \mathrm{~V}\right)$, shows a clockwise hysteresis of the drain current consistent with the accumulation and depletion of $p$-type charge carriers in the channel (Figure 3a). This is consistent with the current retention behavior due to the dipolar polarization of the ferroelectric layer. ${ }^{18}$ In fact, when the polarization switches from one state to another, charges are displaced across the ferroelectric insulator film. The accompanying switching currents are observed as sharp features in the gate current at $-7 \mathrm{~V}$ and at $+7 \mathrm{~V}$, respectively, and as steep increase/decrease of the drain current. Their presence confirms that the memory effect is driven by ferroelectric polarization switching, rather than charge trapping mechanisms or ion migration in and through the ferroelectric layer. ${ }^{20,21}$ Hence, the transistor behaves as a bi-stable memory cell element; before the very first sweep, the ferroelectric film is unpolarized. Upon increasing the negative gate bias beyond the coercive 
field $\left(E_{\mathrm{c}}=53 \mathrm{MV} \mathrm{m}^{-1}\right.$ for the 140-nm-thick $\left.\mathrm{P}(\mathrm{VDF}-\mathrm{TrFE})\right)$ the ferroelectric film becomes fully polarized and an electrical double layer is quickly formed along the ferroelectric/polyelectrolyte interface. At the same time an elevated hole density is established at the semiconductor/polyelectrolyte interface and a high drain current is then measured already at $V_{\mathrm{ds}}$ $=-0.2 \mathrm{~V}$. Upon scanning back the gate voltage, towards and beyond zero, the ferroelectric material is kept polarized and the current remains high (ON-state). At a positive gate voltage bias of around $+7 \mathrm{~V}$, the coercive field is reached and the sign of the ferroelectric polarization changes swiftly. The accumulation of electrons along the semiconductor/polyelectrolyte interface, i.e. in the transistor channel, is severely hampered by the large energy barrier for electron injection from the gold contact into the P3HT conduction band. The output characteristics, $I_{\mathrm{ds}}$ vs. $V_{\mathrm{ds}}$, is shown in Figure $3 \mathrm{~b}$. We note that, at high drain bias voltages where the saturated $I_{\mathrm{ds}}$ is proportional to the square of the gate drive, two distinct slopes are found for $I_{\mathrm{ds}}$ vs. $V_{\mathrm{g}}^{2}$ with a typical crossover point at around $-7 \mathrm{~V}$ (Figure $\mathrm{S} 1$ ). This is consistent with an increased carrier density distribution in the channel region established after that the ferroelectric material becomes polarized. We also notice that the transistor characteristics in fact improve slightly with device ageing, showing excellent performances even after several weeks with readout voltages as low as $V_{\mathrm{ds}}=-50 \mathrm{mV}$ (Figure 3c). This reduced read-out voltage is up to two orders of magnitude lower than what is usually reported for conventional FeFETs. ${ }^{18}$ For comparison, a typical $p$-channel P3HT FeFET with a 200-nm-thick P(VDF-TrFE) gate dielectric without electrolyte interlayer produces output currents no greater than $20 \mathrm{nA}$ at comparable $V_{\mathrm{ds}}{ }^{22}$ We attribute this to the high hygroscopic properties of the P(VPA-AA) layer which allows for an improvement of the impedance characteristics of the ferroelectric/polyelectrolyte/semiconductor configuration $^{23}$ as well as doping of the semiconductor layer. ${ }^{24}$ When the $\mathrm{P}(\mathrm{VDF}-\mathrm{TrFE})$ layer is 
placed in direct contact with P3HT, as in conventional FeFETs, the devices show poor performance at $V_{\mathrm{ds}}=-0.2 \mathrm{~V}$ with $\mathrm{ON} / \mathrm{OFF}$ ratio at zero $V_{\mathrm{g}}$ of about 60 , while no field-effect modulation is observed at even lower $V_{\mathrm{ds}}$ (see Figure $3 \mathrm{~d}$ and Figure S2). This is attributed to the high gate current for these FeFET devices comprising a 140-nm-thick ferroelectric layer, which is of the same order of magnitude as the drain current. The drop in ON/OFF ratio, observed for devices with polyelectrolyte interlayer at high $V_{\mathrm{ds}}(>-1 \mathrm{~V})$, is not due to degradation of the ONcurrent but is due to a shift of the threshold voltage at more positive $V_{\mathrm{g}}$, which thus reduces the final ON/OFF ratio when measured at zero $V_{\mathrm{g}}$.
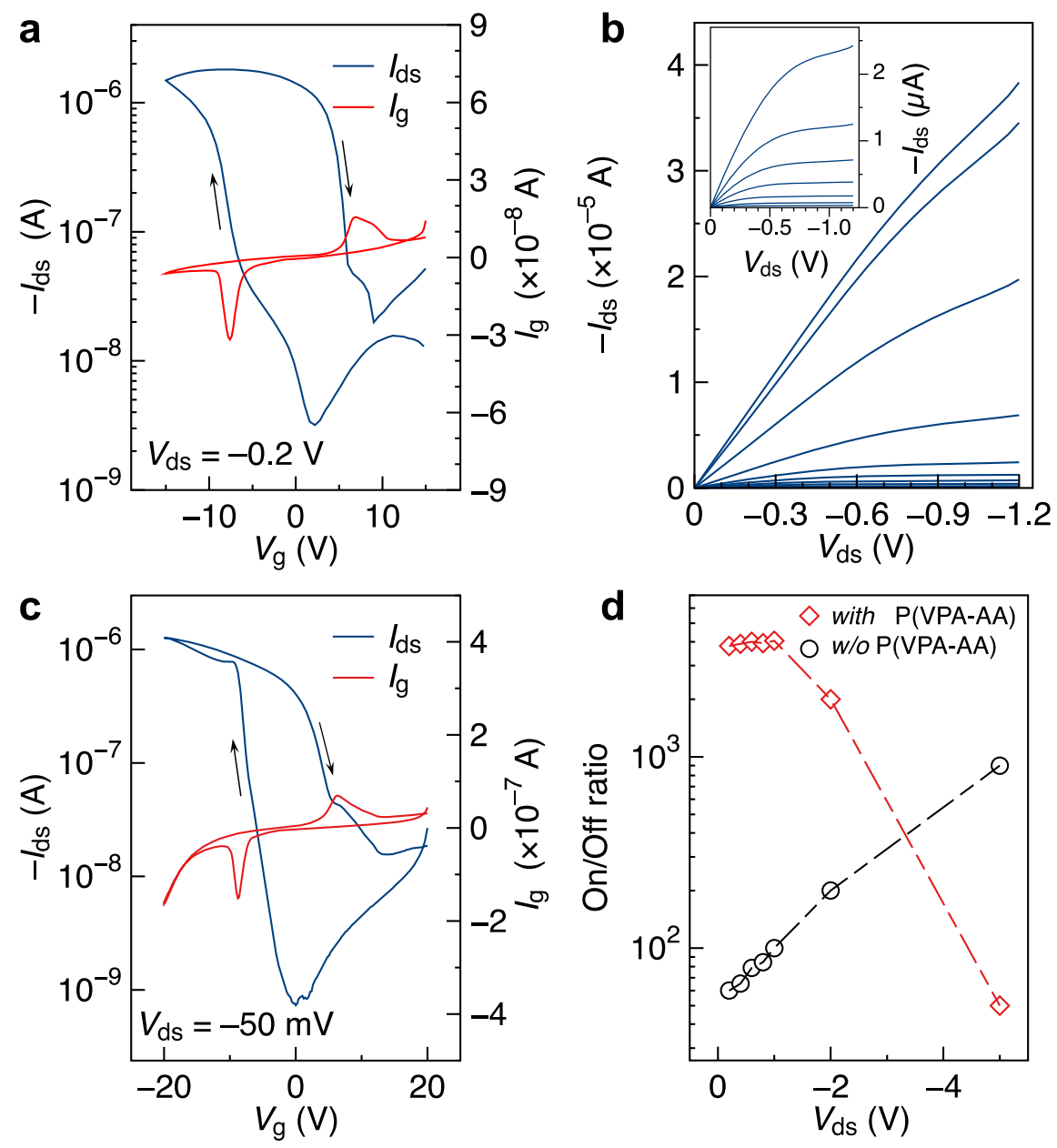
Figure 3. (a) $I_{\mathrm{ds}}$ and $I_{\mathrm{g}}$ as a function of $V_{\mathrm{g}}$ for the P3HT ferroelectric transistor $\left(V_{\mathrm{ds}}=-0.2 \mathrm{~V}\right)$. The arrows show the clockwise hysteresis of the drain current consistent with accumulation and depletion of the charge carriers. (b) $I_{\mathrm{ds}}$ as a function of $V_{\mathrm{ds}}$ for a typical ferroelectric transistor $\left(V_{\mathrm{g}}\right.$ from 0 to $-10 \mathrm{~V}$ in steps of $1 \mathrm{~V}$ ). The inset shows the same data in (b) for $V_{\mathrm{g}}$ from 0 to $-6 \mathrm{~V}$ in steps of $1 \mathrm{~V}$. (c) $I_{\mathrm{ds}}$ and $I_{\mathrm{g}}$ as a function of $V_{\mathrm{g}}$ for a ferroelectric transistor at $V_{\mathrm{ds}}=-50 \mathrm{mV}$. (d) $\mathrm{ON} / \mathrm{OFF}$ ratio as a function of $V_{\mathrm{ds}}$ for ferroelectric OFET with (open squares) and without (open circles) P(VPA-AA) interlayer.

Due to the relatively high coercive field of $\mathrm{P}(\mathrm{VDF}-\mathrm{TrFE})\left(E_{\mathrm{C}} \sim 50 \mathrm{MV} \mathrm{m}^{-1}\right)$, the $V_{\mathrm{g}}$ necessary to switch the devices is rather high. Low-voltage switching transistors can be attained by reducing the ferroelectric layer thickness well below $100 \mathrm{~nm}$. Indeed, by shrinking the P(VDFTrFE) layer thickness down to $60 \mathrm{~nm}$ results in a lower operating bias for switching $\left(V_{\mathrm{C}}<3 \mathrm{~V}\right.$, Figure 4). However, we notice that such a narrow voltage window leads to unstable performance in terms of remanent polarization characteristics. Since the leakage current appears to be independent of the ferroelectric layer thickness (see Figure 3a and Figure 4 for comparison), the primary mechanism of current degradation may be ascribed to a ferroelectric domain pinning due to ionic contaminations which becomes predominant when the ferroelectric layer thickness is lowered down to 50-60 nm. This would result in a low current retention. ${ }^{25}$ 


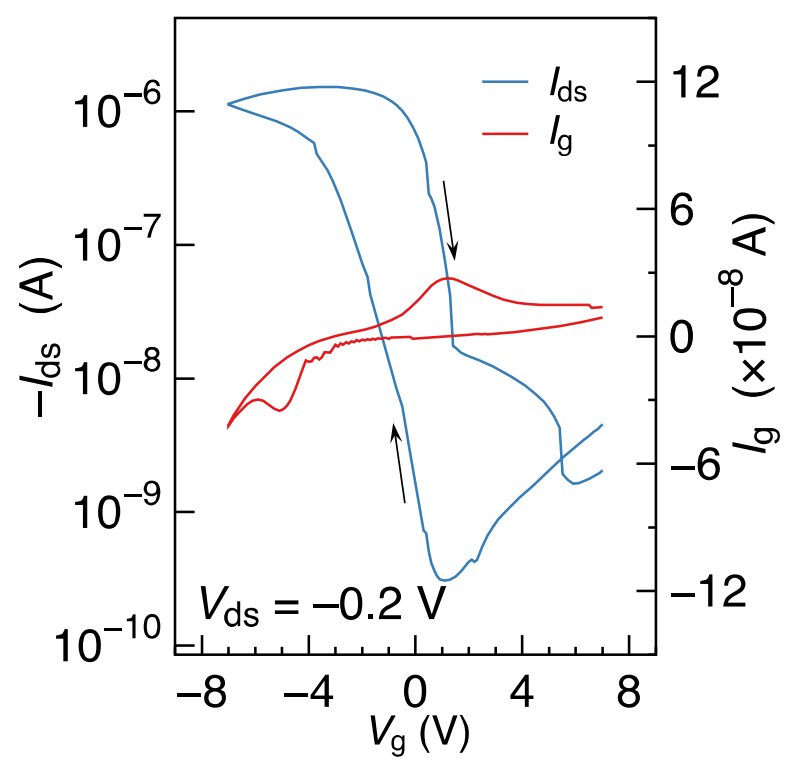

Figure 4. $I_{\mathrm{ds}}$ and $I_{\mathrm{g}}$ as a function of $V_{\mathrm{g}}$ for a P3HT ferroelectric transistor $\left(V_{\mathrm{ds}}=-0.2 \mathrm{~V}\right)$ comprising a 60-nm-thick $\mathrm{P}(\mathrm{VDF}-\mathrm{TrFE})$ layer.

The switching current in our polyelectrolyte-gated transistors was monitored during the experiments using a $5 \mathrm{k} \Omega$ resistor connected in series with the source electrode (Figure 5a). When $V_{\mathrm{ds}}=-0.2 \mathrm{~V}$, the output source-drain current tracks the input gate signal. Distortion of the output waveform is due to short-lived capacitive displacement, which is typical of electrolytegated transistors. ${ }^{8,9}$ When $V_{\mathrm{ds}}=-0.2 \mathrm{~V}$, no steady output current in produced as expected. Interestingly, the OFF-to-ON state transition typically amounts to $\sim 0.2 \mathrm{~ms}$ for pulses of $\pm 10 \mathrm{~V}$. Programming pulses shorter than $0.1 \mathrm{~ms}$ resulted in a state with a low ON-current of the same order of magnitude as the OFF-current. On the other hand, the ON-to-OFF state transition is found to occur in nearly $50 \mu \mathrm{s}$, indicating that the programming time is primarily determined by the conductance of the semiconductor channel. Hence, the switching time is comparable with that of conventional ferroelectric transistors and capacitors where dipoles fully switch in $0.1-0.3$ 
$\mathrm{ms},{ }^{18}$ suggesting that in our devices the programming time is not limited nor affected by the polyelectrolyte interlayer. We note indeed that the typical switching time of electrolyte-gated FETs is also of the order of $0.1 \mathrm{~ms}$ and decreases when the ion mobility is increased (i.e. at higher relative humidity). ${ }^{26}$ The field-effect mobility in the linear regime, $\mu_{\text {lin }}$, has been calculated according to $\mu_{\operatorname{lin}}=\left(\mathrm{LW}^{-1} C_{\mathrm{i}}^{-1} \mathrm{~V}^{-1}\right)\left(d I_{\mathrm{ds}} / d V_{\mathrm{g}}\right)$. The mobility reaches about $0.1 \mathrm{~cm}^{2} \mathrm{~V}^{-1}$ $\mathrm{s}^{-1}$, which is in good agreement with previous reports. ${ }^{27}$ The current levels in the OFF-state and ON-state (the ON/OFF ratio at zero $V_{\mathrm{g}}$ ) differ by 3 orders of magnitude or higher for all the prepared devices.
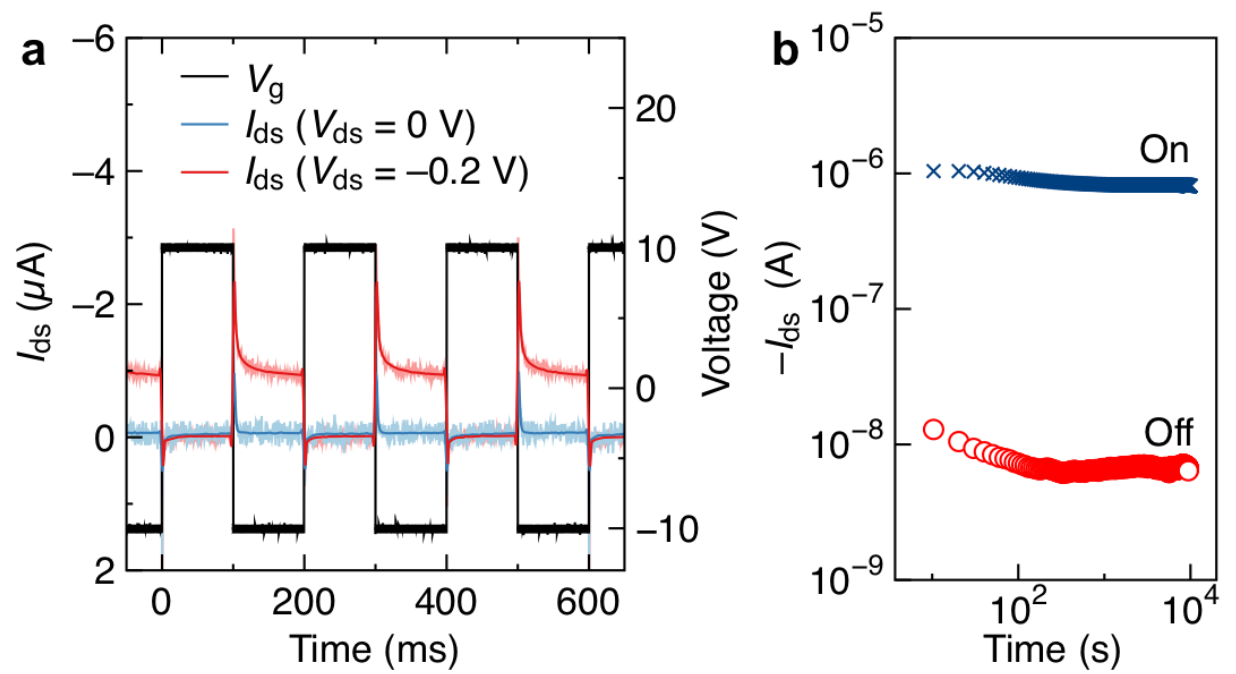

Figure 5. (a) Transient response of a polyelectrolyte-gated ferroelectric transistor at constant $V_{\mathrm{ds}}$ when the $V_{\mathrm{g}}$ is pulsed at $10 \mathrm{~Hz}$. (b) Data retention time obtained by programming the device once in the ON- or OFF-state and monitoring the drain current over time at $V_{\mathrm{ds}}=-0.2 \mathrm{~V}$ and zero gate bias.

Data retention characteristics were examined by measuring the remnant drain current level as a function of time. Long retention time is typical for ferroelectric capacitors ${ }^{17}$ and FETs. ${ }^{18}$ Figure 
$5 \mathrm{~b}$ shows the value of drain current in the $\mathrm{ON}$ - and OFF-states as a function of the retention time, measured at $V_{\mathrm{ds}}=-0.2 \mathrm{~V}$. The $\mathrm{ON}$ - and OFF-states were programmed by poling the ferroelectric at gate voltages of $-15 \mathrm{~V}$ and $+15 \mathrm{~V}$ with a $3 \mathrm{~s}$ pulse, respectively. The memory retention is found to be rather excellent for these devices with the ON/OFF ratio being more than two orders

of magnitude even after $10^{4} \mathrm{~s}$. The long retention time strengthen the viability of this kind of devices for non-volatile memory applications.

\section{Conclusions}

In summary, we have demonstrated that the surface charge density expressed by a ferroelectric polymer thin film matches the density of charges in a dissociated polyelectrolyte. This ferroelectric-induced electric double layer promotes memory functionality in electrolyte-gated FETs. The combination of ferroelectric and polyelectrolyte bi-layer as the gate insulator provides a large specific capacitance $\left(\sim 1 \mu \mathrm{F} \mathrm{cm}{ }^{-2}\right)$, fast polarization response times $(\sim 0.2 \mathrm{~ms})$ and high semiconductor surface charge density at source-drain voltages of only fractions of a volt, resulting in non-volatile memory devices. Notably, this ferroelectric-induced EDL shows long retention times of more than $10^{4} \mathrm{~s}$.

\section{Experimental methods}

The ferroelectric P(VDF-TrFE) 70/30 mol\% copolymer was purchased from Solvay SA and used as received. The $\mathrm{P}(\mathrm{VDF}-\mathrm{TrFE})$ powder was dissolved in diethyl carbonate (DEC) at a concentration of $4 \% \mathrm{w} / \mathrm{v}$ and filtered through a $0.1 \mu \mathrm{m}$ filter. The polyelectrolyte P(VPA-AA)) was supplied by Rhodia, dissolved in a mixture of 1-propanol and deionized water (4\% w/v) with a solvent ratio of $4: 1$ and filtered with a $0.2 \mu \mathrm{m}$ nylon syringe filter. Regioregular poly(3- 
hexylthiophene) (P3HT) was purchased from Sigma-Aldrich and used without further purification. The semiconductor was dissolved in 1,2-dichlorobenzene $(1 \% \mathrm{w} / \mathrm{v})$ and filtered with a $0.2 \mu \mathrm{m}$ polytetrafluoroethylene (PTFE) syringe filter. Thin films were prepared by spincoating in a class 1,000 clean room environment.

Corning glass substrates were cleaned sequentially in deionized water, acetone and isopropanol. Interdigitated source and drain electrode (3-nm-thick Ti and 27-nm-thick Au) were defined by photolithography and wet etching procedure. The substrates were cleaned carefully again using deionized water, acetone and isopropanol before use. The semiconductor layer was formed by spin-coating the warm solution at $2000 \mathrm{rpm}$ for $30 \mathrm{~s}$ giving a film thickness of $30 \mathrm{~nm}$. The films were then annealed at $120^{\circ} \mathrm{C}$ for 30 min under nitrogen. The polyelectrolyte solution was spin-coated at $2000 \mathrm{rpm}$ for $60 \mathrm{~s}$ and dried on a hot plate under vacuum at $120^{\circ} \mathrm{C}$ for $120 \mathrm{~s}$, resulting in a thickness of about $120 \mathrm{~nm}$. The $\mathrm{P}(\mathrm{VDF}-\mathrm{TrFE})$ layer was subsequently spin-coated on top of the polyelectrolyte layer and annealed at $135^{\circ} \mathrm{C}$ for $1 \mathrm{~h}$ in vacuum oven in order to increase the film crystallinity. The film thickness was controlled by varying the spin speed, measured by ellipsometry and confirmed by using a DekTak profilometer. An 80-nm-thick top electrode for the capacitors and gate electrode for the transistors are formed by thermal evaporation of various metals through a Ni shadow mask (Tecan Ltd.).

The electrical characteristics of the ferroelectric transistors were measured using a semiconductor parameter analyzer (Keithley 4200-SCS). The impedance measurements were carried out with an Alpha high-resolution dielectric analyzer (Novocontrol GmbH). An AC voltage of $0.3 \mathrm{~V}$ was applied, the frequency was set at $1 \mathrm{kHz}$, and the DC voltage was swept from positive to negative voltages. An equivalent circuit model made of a resistor and a capacitor 
in parallel was used to extract the effective capacitance, which was calculated from the equation $C=1 /(2 \pi f \operatorname{Im}(Z))$ and where $f$ is the frequency and $Z$ is the measured impedance.

\section{AUTHOR INFORMATION}

\section{Corresponding Author}

*E-mail: simone.fabiano@liu.se

\section{ACKNOWLEDGMENT}

This research was partially supported by the Advanced Functional Materials Center at Linköping University and the Önnesjö Foundation. Authors wish to thank also the Knut and Alice

Wallenberg Foundation (Power Paper project, scholars) and VINNOVA for financial support.

\section{ASSOCIATED CONTENT}

Supporting Information Available: Electrical measurements, Figures S1-S2. This material is available free of charge via the Internet at http://pubs.acs.org.

\section{REFERENCES}

(1) Scott, J. F.; Dearaujo, C. A. P. Science 1989, 246, 1400-1405.

(2) Berggren, M.; Nilsson, D.; Robinson, N. D. Nat. Mater. 2007, 6, 3-5.

(3) Scott, J. C.; Bozano, L. D. Adv. Mater. 2007, 19, 1452-1463.

(4) Lovinger, A. J. Science 1983, 220, 1115-1121.

(5) Horiuchi, S.; Tokura, Y. Nat. Mater. 2008, 7, 357-366.

(6) Li, M.; Wondergem, H. J.; Spijkman, M.-J.; Asadi, K.; Katsouras, I.; Blom, P. W. M.; de Leeuw, D. M. Nat. Mater. 2013, 12, 433-438. 
(7) Dhoot, A. S.; Yuen, J. D.; Heeney, M.; McCulloch, I.; Moses, D.; Heeger, A. J. PNAS 2006, 103, 11834-11837.

(8) Cho, J. H.; Lee, J.; Xia, Y.; Kim, B.; He, Y. Y.; Renn, M. J.; Lodge, T. P.; Frisbie, C. D. Nat. Mater. 2008, 7, 900-906.

(9) Herlogsson, L.; Crispin, X.; Robinson, N. D.; Sandberg, M.; Hagel, O. J.; Gustafsson, G.; Berggren, M. Adv. Mater. 2007, 19, 97-101.

(10) Naber, R. C. G.; Blom, P. W. M.; Marsman, A. W.; de Leeuw, D. M. Appl. Phys. Lett. 2004, 85, 2032.

(11) Ferris, R. J.; Lin, S. H.; Therezien, M.; Yellen, B. B.; Zauscher, S. Acs Appl. Mater. Interfaces 2013, 5, 2610-2617.

(12) Ferris, R.; Yellen, B.; Zauscher, S. Small 2012, 8, 28-35.

(13) Wang, F. P.; Lack, A.; Xie, Z. L.; Frubing, P.; Taubert, A.; Gerhard, R. Appl. Phys. Lett. 2012, 100, 062903.

(14) Braun, S.; Salaneck, W. R.; Fahlman, M. Adv. Mater. 2009, 21, 1450-1472.

(15) Naber, R. C. G.; Massolt, J.; Spijkman, M.; Asadi, K.; Blom, P. W. M.; de Leeuw, D. M. Appl. Phys. Lett. 2007, 90, 113509.

(16) Kalbitz, R.; Frübing, P.; Gerhard, R.; Taylor, D. M. Appl. Phys. Lett. 2011, 98, 033303.

(17) Asadi, K.; Leeuw, D. M. D.; Boer, B. D.; Blom, P. W. M. Nat. Mater. 2008, 7, 547-550. 
(18) Naber, R. C. G.; Tanase, C.; Blom, P. W. M.; Gelinck, G. H.; Marsman, A. W.; Touwslager, F. J.; Setayesh, S.; De Leeuw, D. M. Nat. Mater. 2005, 4, 243-248.

(19) Khan, M. A.; Bhansali, U. S.; Alshareef, H. N. Adv. Mater. 2012, 24, 2165-2170.

(20) Sekitani, T.; Yokota, T.; Zschieschang, U.; Klauk, H.; Bauer, S.; Takeuchi, K.; Takamiya, M.; Sakurai, T.; Someya, T. Science 2009, 326, 1516-1519.

(21) Egginger, M.; Irimia-Vladu, M.; Schwodiauer, R.; Tanda, A.; Frischauf, I.; Bauer, S.; Sariciftci, N. S. Adv. Mater. 2008, 20, 1018-1022.

(22) Naber, R. C. G.; de Boer, B.; Blom, P. W. M.; de Leeuw, D. M. Appl. Phys. Lett. 2005, 87, 203509.

(23) Said, E.; Larsson, O.; Berggren, M.; Crispin, X. Adv. Funct. Mater. 2008, 18, 3529-3536.

(24) Fabiano, S.; Braun, S.; Fahlman, M.; Crispin, X.; Berggren, M. Adv. Funct. Mater. [online early access] 2013, DOI: 10.1002/adfm.201302070. Published online: 28 Aug 2013. http://onlinelibrary.wiley.com/doi/10.1002/adfm.201302070/abstract (accessed 28 Aug 2013).

(25) Gelinck, G. H.; Marsman, A. W.; Touwslager, F. J.; Setayesh, S.; de Leeuw, D. M.; Naber, R. C. G.; Blom, P. W. M. Appl. Phys. Lett. 2005, 87, 092903.

(26) Larsson, O.; Said, E.; Berggren, M.; Crispin, X. Adv. Funct. Mater. 2009, 19, 3334-3341.

(27) Naber, R. C. G.; Mulder, M.; de Boer, B.; Blom, P. W. M.; de Leeuw, D. M. Org. Electron. 2006, 7, 132-136. 
TOC

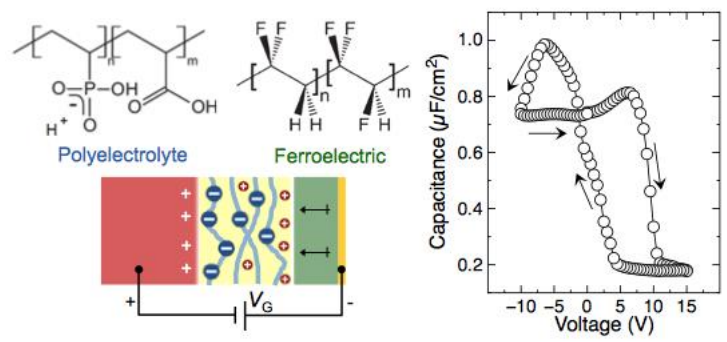




\section{Supporting Information}

Ferroelectric polarization induces electric double layer bistability in electrolyte-gated field-effect transistors

Simone Fabiano, * Xavier Crispin, and Magnus Berggren

Organic Electronics, Department of Science and Technology, Linköping University, SE-601 74, Norrköping (Sweden) E-mail: simone.fabiano@liu.se 


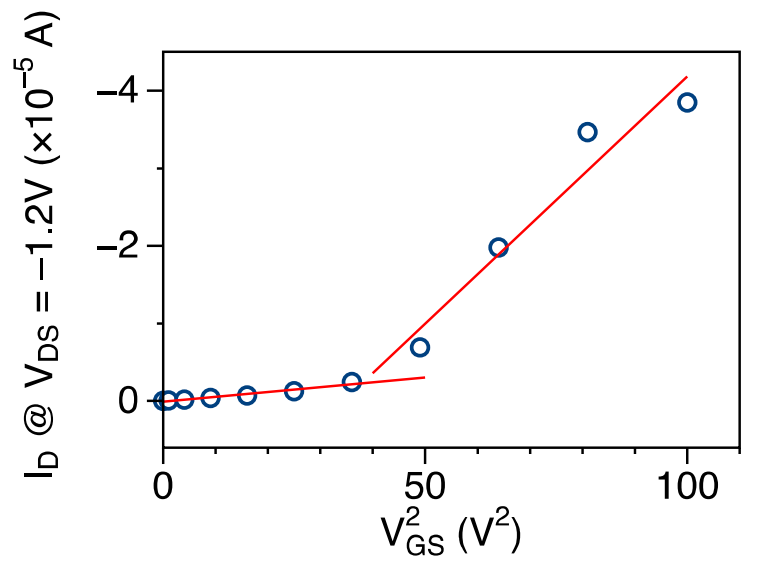

Figure S1 Saturated source-drain current as measured at $V_{\mathrm{ds}}=-1.2 \mathrm{~V}$ as a function of the squared gate voltage for the P3HT p-channel transistor shown in Fig. 2 of the main text. The figure shows two distinct slopes for $I_{\mathrm{ds}}\left(V_{\mathrm{g}}^{2}\right)$ with typical crossover point at around $-7 \mathrm{~V}$. This is consistent with an increased carrier density distribution in the channel region after ferroelectric polarization. 


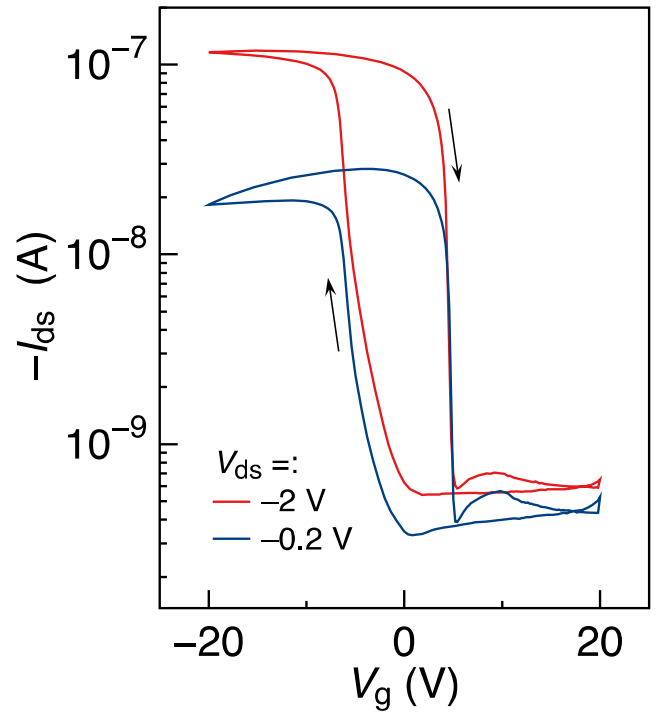

Figure S2 Source-drain current as a function of gate voltage for a conventional P3HT-based ferroelectric transistor where the 140-nm-thick $\mathrm{P}(\mathrm{VDF}-\mathrm{TrFE})$ layer is place in direct contact with the semiconductor layer. The FET has a channel length of $20 \mu \mathrm{m}$ and a channel width of $1,000 \mu \mathrm{m}$. 